\title{
Síndrome de absceso hepático invasor por Klebsiella pneumoniae, serie de casos
}

\author{
Invasive liver abscess syndrome caused by Klebsiella pneumoniae, \\ case series \\ Martín Ignacio Lapidus ${ }^{1}$, Melisa Altavista ${ }^{1}$, Marco Gornatti ${ }^{1}$ André Falcón' ${ }^{1}$, Marina Alonso Serena ${ }^{2}$ y María Belén Bonella
}

'Servicio de Clínica Médica, Hospital Italiano de Buenos Aires. Buenos Aires, Argentina.

${ }^{2}$ Área de Investigación en Medicina Interna. Hospital Italiano de Buenos Aires. Buenos Aires, Argentina.

Los autores declaran no tener conflictos de interés.

Los autores no han recibido fuente de financiamiento alguna para la realización del estudio.

Recibido (segunda versión): 11 de agosto de 2020 / Aceptado: 31 de agosto de 2020

\section{Resumen}

Introducción: Los abscesos hepáticos primarios producidos por Klebsiella pneumoniae y las implicancias sistémicas relacionadas constituyen el síndrome de absceso hepático invasor por Klebsiella pneumoniae. Objetivo: Describir las características clínicas, epidemiológicas y la evolución de esta entidad en nuestro centro. Pacientes $y$ Métodos: Cohorte retrospectiva de pacientes adultos internados en el Hospital Italiano de Buenos aires entre el 1 de enero de 2001 y el 1 de mayo de 2020. Se incluyeron pacientes con diagnóstico de absceso en cualquier órgano con aislamiento en cultivo de absceso o hemocultivo positivo para Klebsiella pneumoniae para ser analizadas las características epidemiológicas, clínicas y la evolución de aquellos con localización primaria hepática. Resultados: 10 pacientes fueron incluidos. Dos $(20 \%)$ pacientes fueron orientales. La mediana de edad fue 69 años (IIC 64-79), nueve (90\%) fueron hombres. La comorbilidad más frecuente fue diabetes mellitus tipo 2 (40\%). Cuatro (40\%) pacientes tuvieron diseminación a otros órganos. La mediana de internación fue 21,5 días (IIC 15-43), 60\% (n: 6) requirió internación en unidad de cuidados intensivos y $30 \%$ (n: 3 ) de los pacientes falleció. Conclusión: El síndrome de absceso hepático invasor por Klebsiella pneumoniae es una enfermedad infrecuente, potencialmente mortal. Este estudio intenta reportar las características de los pacientes con esta patología en nuestra población.

Palabras clave: Klebsiella pneumoniae; absceso hepático; sepsis.

\section{Abstract}

Background: Primary liver abscesses caused by Klebsiella pneumoniae and their related systemic complications produce the invasive liver abscess syndrome due to Klebsiella pneumoniae. Aim: To describe the clinical, epidemiological and evolution characteristics in our center. Methods: A retrospective cohort of hospitalized adults in Hospital Italiano de Buenos Aires between January 1st, 2001 and May 1st, 2020. We included patients with diagnosis of abscess in any organ with rescue in culture or positive blood culture for Klebsiella pneumoniae. Epidemiological, clinical characteristics and prognosis of those with hepatic primary localization were analyzed. Results: 10 patients were included. Two (20\%) patients were Oriental. Median age was 69 years (interquartile range $64-79)$, nine $(90 \%)$ were men. The most frequent comorbidity was type 2 diabetes $(40 \%)$. Four $(40 \%)$ patients had spread to other organs. The median hospitalization was 21.5 days (IIC 15-43), 60\% (n: 6) were hospitalized in the intensive care unit and $30 \%$ (n: 3 ) died. Conclusions: The invasive liver abscess syndrome due to Klebsiella pneumoniae is a rare life-threatening disease. Our study reports the characteristics of patients with this syndrome in our population.

Keywords: Klebsiella pneumoniae; liver abscess; pyogenic liver abscess; sepsis 
funciona como centro de derivación nacional. Cuenta con un repositorio único de información de cada paciente. Este repositorio se centraliza a través de una historia clínica electrónica (HCE) orientada a problemas. Los problemas, diagnósticos, antecedentes, medicación, procedimientos, estudios e información administrativa, entre otros, se almacenan y codifican utilizando vocabulario controlado.

Como estrategia de cribado para la detección de los pacientes potencialmente incluibles se generó una lista utilizando vocabulario controlado de todos los pacientes adultos mayores a 18 años que tuvieran cargado el término absceso por Klebsiella sp o alguno de los sinónimos, entre los problemas, diagnósticos principales o secundarios o cualquier campo de la epicrisis. Para aumentar la sensibilidad de la búsqueda, se solicitó el listado de pacientes con cultivos positivos para $K$. pneumoniae y el listado de pacientes con cualquier estudio por imagen durante la internación. Se evaluaron los episodios pre-quirúrgicos de los pacientes en quienes se realizara procedimientos percutáneos.

Cuatro médicos entrenados revisaron las HCE de los pacientes detectados y definieron los casos de absceso hepático primario por K. pneumoniae. Se revisaron los criterios de inclusión de: edad igual o mayor a 18 años, pacientes con presencia de abscesos en cualquier órgano diagnosticado por imagen o por punción percutánea o drenaje quirúrgico y pacientes con aislamiento en cultivo de absceso o hemocultivos, de $K$. pneumoniae. Se excluyeron a las pacientes embarazadas, portadores de enfermedad hepato-biliar (cirrosis, colangitis, colocación de stent biliar o cirugía hepato-biliar) y enfermedad colo-rectal (cáncer de colon, cáncer de recto, cáncer colorectal, cirugía por cáncer de colon o cirugía por cáncer de recto). Del listado de casos, se evaluó las características clínicas y de laboratorio. Se decidió excluir a pacientes con antecedente de patología hepato-biliar y colo-rectal para seleccionar a los pacientes con absceso primario y obtener una población similar a la serie publicada por Wang y cols ${ }^{7}$.

Se presentan las variables categóricas como porcentajes y las variables continuas como media y desvío estándar (DE) o medianas e intervalos intercuartílicos (IIC) según distribución observada. El análisis estadístico se realizó con el software STATA versión 14.0.

\section{Resultados}

Durante el período evaluado se detectaron 54 potenciales casos, se excluyeron 44 pacientes por cumplir con criterios de exclusión. Se analizaron 10 pacientes con diagnóstico de absceso primario hepático por $K$. pneumoniae.

La media de edad fue 69 años (IIC 64-79), nueve (90\%)
El HIBA es un hospital universitario de alta complejidad de la ciudad autónoma de Buenos Aires, que por $K$ pneumoniae internados en el Hospital Italiano de Buenos Aires (HIBA) entre el 1 de enero de 2001 y el 1 de mayo de 2020. Se definió absceso primario hepático a producido en ausencia de enfermedad predisponente hepato-biliar o colo-rectal, en concordancia con la definición propuesta por Wen-Liang Yu en su revisión del síndrome ${ }^{1}$. El estudio fue evaluado y aprobado por el comité institucional de ética de protocolos de investigación. 
eran hombres, dos $(20 \%)$ pacientes eran orientales. La comorbilidad más frecuente fue diabetes mellitus en cuatro (40\%) pacientes, seguida de enfermedad oncológica activa en tres (30\%) y esteatosis hepática en tres (30\%) (Tabla 1).

En el laboratorio general, sólo tres pacientes tuvieron medida la proteína $\mathrm{C}$ reactiva (PCR), con una mediana de PCR de 293 mg/L (rango interquartil 203-532). Todos los pacientes tuvieron medido un recuento de leucocitos, con una mediana de $17.585 / \mathrm{mm}^{3}$ (rango interquartil 12.240-21.030).

Cuatro (40\%) pacientes tuvieron diseminación a otras localizaciones, siendo la más frecuente el líquido pleural, en cuatro (40\%) casos (Tabla 2). Cinco (50\%) de los pacientes tuvieron hemocultivos positivos con aislamiento de K. pneumoniae. Nueve (90\%) pacientes tuvieron aislamiento de $K$. pneumoniae en el absceso, cuatro (40\%) pacientes tuvieron aislamiento de $K$. pneumoniae en el líquido pleural.

En nueve (90\%) de los pacientes se aisló una cepa de $K$. pneumoniae resistente a ampicilina, sensible a ampicilina/sulbactam, cefalosporina de tercera generación y carbapenémicos. Hubo un solo caso de $K$. pneumoniae con resistencia a cefalosporinas, sensibilidad disminuida a carbapenémicos y sensible a amikacina.

Todos los pacientes recibieron drenaje de abscesos y antibioterapia efectiva según antibiograma. Con respecto a la evolución de los pacientes la mediana de internación fue 21,5 días (rango intercuartil 15-43). Seis (60\%) de los pacientes requirieron, en algún momento de la estadía hospitalaria, internación en unidad de cuidados intensivos y tres (30\%) fallecieron dentro de los 90 días de la internación.

\section{Discusión}

El síndrome de absceso hepático invasor por $K$. pneumoniae es una entidad infrecuente, potencialmente letal. Ésta se ha reportado en series de casos de países asiáticos como Taiwán, Corea y China ${ }^{3}$. Sin embargo, en los últimos años han aumentado los reportes y las series de casos de absceso hepático en países de occidente, como Estados Unidos de América y Chile, aunque en muchos de estos casos, los abscesos no eran exclusivamente producidos por $K$. pneumoniae $e^{8,9}$. En nuestro estudio describimos las características epidemiológicas, clínicas y la evolución de los pacientes con diagnóstico de absceso hepático por $K$. pneumoniae, haciendo foco en aquellos que, por ausencia de factores predisponentes, presumimos que cursaron el síndrome de absceso hepático invasor por $K$. pneumoniae, en un centro en Latinoamérica.

Con respecto a las características epidemiológicas, en nuestro estudio observamos que dos pacientes fueron orientales, dato que resulta de interesante mención
Tabla 1. Características de la población de pacientes con absceso hepático por Klebsiella pneumoniae $(n=10)$

\begin{tabular}{ll}
\hline Edad media (años), (mediana) & 69 (IIC 64-79) \\
\hline Hombres & $9(90 \%)$ \\
Orientales & $2(20 \%)$ \\
Co-morbilidades & \\
- Enfermedad respiratoria & $1(10 \%)$ \\
- Diabetes mellitus & $4(40 \%)$ \\
- Insuficiencia cardiaca & - \\
- Insuficiencia renal crónica & $1(10 \%)$ \\
- Enfermedades oncológicas & $3(30 \%)$ \\
- VIH/SIDA & - \\
- Enfermedades autoinmunes & - \\
- Esteatosis hepática & $3(30 \%)$ \\
- Alcoholismo & - \\
\hline
\end{tabular}

Tabla 2. Localización más frecuente de los abscesos causados por Klebsiella pneumoniae

Sitio de localización de abscesos $\quad \mathrm{n}=10$

Hepático $10(100 \%)$

Cavidad pleural $\quad 4(40 \%)$

Oftálmico

$2(20 \%)$

Pulmonar

$1(10 \%)$

Otros órganos

$2(20 \%)$

teniendo en cuenta que la muestra se obtuvo a partir del registro del HIBA con población occidental. Esto refuerza el concepto de que esta patología es de mayor prevalencia en países orientales, donde fue reconocida y descrita por primera vez. Sin embargo, no es claro si los descendientes asiáticos tienen un riesgo genético para estas infecciones o si esta bacteria es de mayor prevalencia en países asiáticos ${ }^{10}$. Un estudio que evaluó aislados bacterianos en muestras de materia fecal de individuos sanos de distintos países asiáticos (China, Taiwan, Japón, entre otros) reportó $10 \%$ de colonización por $K$. pneumoniae $\mathrm{K} 1 / \mathrm{K} 2^{11}$.

La media de edad reportada en nuestro estudio fue mayor en comparación a lo reportado en otras series ${ }^{1}$. En concordancia con los estudios previos, la co-morbilidad más frecuente fue diabetes mellitus tipo $2^{2}$. Es conocido que la diabetes mellitus interfiere con la quimiotaxis y fagocitosis de los neutrófilos; sin embargo, esta influencia en la función de los macrófagos es todavía desconocida ${ }^{2}$. Estudios identificaron el mal control glucémico como factor de riesgo para susceptibilidad a serotipos K1/K2 en pacientes con absceso hepático por $K$. pneumoniae y endoftalmitis complicada ${ }^{5}$. 
de letalidad ${ }^{3}$. En nuestro estudio se reportó una letalidad de $30 \%$ (3/10) dentro de los 90 días de la internación índice.

\section{Conclusión}

En comparación a las series orientales, en nuestro estudio se observó un curso más agresivo de la enfermedad con mayor incidencia de enfermedad diseminada y mayor letalidad. El reconocimiento temprano del síndrome con la correspondiente evaluación multisistémica y tratamiento oportuno orientado a drenar los focos abscedados y asegurar la antibioterapia efectiva, podría mejorar los desenlaces clínicos en nuestro medio. Consideramos que un mayor estudio epidemiológico y clínico sobre esta infección permitirá una mejor comprensión y tratamiento de la misma.

\section{Referencias bibliográficas}

1.- $\quad$ Yu W-L M D. Invasive liver abscess syndrome caused by Klebsiella pneumoniae. Post TW, ed. UpToDate. Waltham, MA: UpToDate Inc. https://www.uptodate.com (revisado en julio de 2020).

2.- Fung C-P, Chang F-Y, Lee S-C, Hu B-S, Kuo BI-T, Liu C-Y, et al. A global emerging disease of Klebsiella pneumoniae liver abscess: is serotype $\mathrm{K} 1$ an important factor for complicated endophthalmitis? Gut 2002; 50: 420-4. doi: 10.1136/gut.50.3.420.

3.- Fang C-T, Fang C T, Lai S Y, Yi W C, Hsueh P R, Liu K-L, et al. Klebsiella pneumoniae genotype K1: an emerging pathogen that causes septic ocular or central nervous system complications from pyogenic liver abscess. Clin Infect Dis 2007; 45: 284-93. https://doi. org/10.1086/519262.

4.- $\quad$ Lin J-C, Chang F-Y, Fung C-P, Xu J-Z, Cheng H-P, Wang J-J, et al. High prevalence of phagocytic-resistant capsular serotypes of Klebsiella pneumoniae in liver abscess. Microbes Infect 2004; 6: 1191-8. doi: 10.1016/j.micinf.2004.06.003.

5.- Lin J-C, Siu LK, Fung C-P, Tsou H-H, Wang J-J, Chen C-T, et al. Impaired phagocytosis of capsular serotypes K1 or K2 Klebsiella pneumoniae in type 2 diabetes mellitus patients with poor glycemic control. J Clin Endocrinol Metab 2006; 91: 3084-7. doi: 10.1210/jc.20052749.

6.- $\quad$ Li J, Fu Y, Wang J-Y, Tu C-T, Shen X-Z, Li L, et al. Early diagnosis and therapeutic choice of Klebsiella pneumoniae liver abscess. Front Med China 2010; 4 (3): 308-16. doi: 10.1007/ s11684-010-0103-9.

7.- Wang J H, Liu Y C, Lee S S, Yen M Y, Chen Y $\mathrm{S}$, Wang J H, et al. Primary liver abscess due to Klebsiella pneumoniae in Taiwan. Clin. Infect. Dis 1998; 26: 1434-8. doi: 10.1086/516369.

8.- Fantuzzi S A, Albertz A N, Valenzuela V A, Estuardo A N, Castro L A. Hepatic abscess: series of 107 cases and literature review. Rev Chilena Infectol 2009; 26: 49-53. http://dx.doi. org/10.4067/S0716-10182009000100007.

9.- Rahimian J, Wilson T, Oram V, Holzman R S. Pyogenic liver abscess: recent trends in etiology and mortality. Clin Infect Dis 2004; 39: 1654-9. doi: $10.1086 / 425616$.

10.- Mgbemena O, Serota D P, Kumar S, Wozniak J E, Weiss D S, Kempker R R. Peculiar purulence: hypervirulent Klebsiella pneumoniae causing pyomyositis. Int J Infect Dis 2017; 65: 90-2. doi: 10.1016/j.ijid.2017.09.030.

11.- Lin, Y-T, Siu L K, Lin J-C, Chen T-L,Tseng C-P, Yeh K-M, et al. Seroepidemiology of Klebsiella pneumoniae colonizing the intestinal tract of healthy Chinese and overseas Chinese adults in Asian countries. BMC Microbiol 2012; 12: 13 doi: 10.1186/1471-2180-12-13.

12.- Oikonomou K G, Aye M. Klebsiella pneumoniae liver abscess: a case series of six Asian patients. Am J Case Rep. 2017; 18: 102833. doi: 10.12659/AJCR.905191. 